\title{
TOR kinase controls shoot development by translational regulation of cytokinin catabolic enzymes
}

\section{Denis Janocha', Anne Pfeiffer, ${ }^{1,2}$, Yihan Dong ${ }^{3}$, Ondřej Novák ${ }^{4}$, Miroslav Strnad", Lyuba A Ryabova ${ }^{3}$ \& Jan U. Lohmann ${ }^{1 *}$}

1Department of Stem Cell Biology, Centre for Organismal Studies, Heidelberg University; D-69120 Heidelberg, Germany

${ }^{2}$ current address: Bayer AG, Crop Science Division, Alfred-Nobel-Str. 50, 40789 Monheim am Rhein, Germany

${ }^{3}$ Institut de biologie de moléculaire des plantes UPR2357 du CNRS, Université de Strasbourg, Strasbourg, France

${ }^{4}$ Laboratory of Growth Regulators, Institute of Experimental Botany, The Czech Academy of Sciences and Faculty of Science, Palacký University, Šlechtitelů 27, 78371, Olomouc, Czech Republic

${ }^{*}$ corresponding author

Mailing address of corresponding author:

Jan U. Lohmann

Centre for Organismal Studies

Department of Stem Cell Biology

Heidelberg University

Im Neuenheimer Feld 230

$\mathrm{PH}:+496221546269$

D-69120 Heidelberg

FX: +496221546424

Germany

EM:jan.lohmann@cos.uni-heidelberg.de 


\begin{abstract}
Plants continuously adjust the rate at which new organs are produced in accordance with endogenous and environmental signals. Therefore, a multitude of signaling pathways converge to modulate stem cell activity. We have shown previously, that the TOR kinase network integrates metabolic- and light signals to control expression of WUSCHEL, a transcriptional master regulator of stem cells in the shoot apical meristem (SAM). However, the link between TOR and WUS promoter activity remained unresolved. Here we demonstrate that TOR controls trans-Zeatin abundance, the cytokinin derivative that is the main driver of shoot development. Moreover, we identify TOR mediated translational control of cytokinin degrading CYTOKININ OXIDASES/DEHYDROGENASE (CKX) enzymes as the underlying mechanism, which allows the plant to adjust the stem cell signaling environment and growth pattern within minutes after changes in environmental parameters.
\end{abstract}




\section{Introduction}

Due to their sessile life-style, plants depend on their ability to dynamically adjust organ development and growth rates in response to variable environmental conditions. Therefore, they are able to integrate diverse local and systemic signals to adjust stem cell behavior accordingly. Stem cells of the shoot apical meristem (SAM) are controlled by the activity of the homeodomain transcription factor WUSCHEL (WUS) (Bäurle \& Laux, 2005; Daum et al., 2014; Laux et al., 1996; Mayer et al., 1998; Schoof et al., 2000) and previous studies have shown that WUS expression is modulated in response to environmental signals such as nitrate availability or the current light- and energy regime (Janocha \& Lohmann, 2018; Landrein et al., 2018; Li et al., 2017; Pfeiffer et al., 2016). In the absence of a neuronal system, plants make use of complex hormone signaling systems for long distance information relay. Among those, cytokinins (CKs) and particularly trans-Zeatin ( $t Z)$, are involved in nitrate and light signaling from root and leaves to the SAM, respectively (Landrein et al., 2018; Yoshida et al., 2011). CKs in turn promote WUS expression, thus tuning stem cell number in response to the environment (Buechel et al., 2010; Gordon et al., 2009; Kiba et al., 2013; Landrein et al., 2018; Osugi et al., 2017).

CK homeostasis is controlled by tightly regulated biosynthesis and degradation systems (Heyl et al., 2018), with ISOPENTYLTRANSFERASES (IPTs) catalyzing the first step in the anabolic pathway. IPT enzymes synthesize isopentyladenosine-5'-monophosphate (iPRMP) precursors, which can be hydroxylated by CYP735A1/2 to obtain trans-Zeatin riboside-5'monophosphate (tZRMP). Both precursors are then converted by so called LONELY GUY (LOGs) enzymes to obtain the active nucleobases isopentyladenin (iP) and $t Z$ (Kuroha et al., 2009). CK degradation in Arabidopsis is mediated by seven CYTOKININ DEHYDROGENASE/ OXIDASE (CKX) enzymes, which are important players to control CK signaling (Bartrina et al., 2011; Holst et al., 2011; Köllmer et al., 2014; X. Wang et al., 2020). Intracellular CK signal transduction is based on a two-component phospho-relay (Kieber \& Schaller, 2014). The receptors are ARABIDOPSIS HISTIDINE KINASES (AHKs) and reside on the plasma membrane and the endoplasmic reticulum (Antoniadi et al., 2020). Upon CK binding, AHKs first auto-phosphorylate and then pass on the phosphoryl group to ARABIDOPSIS HISTIDINE PHOPHOTRANSFER PROTEINS (AHPS). AHPs continuously shuttle between cytoplasm and nucleus where they interact with and activate type-B ARABIDOPSIS RESPONSE REGULATORS (ARRs) transcription factors by phosphorylation (Kieber \& Schaller, 2014). These regulators induce expression of CK response genes, including type-A ARRs, which in turn dampen the CK response and serve as indicators for $\mathrm{CK}$ 
pathway activation. In addition, several genes encoding enzymes involved in CK biosynthesis and degradation are among the CK response genes, resulting in complex, tissue specific feedback systems.

While continuously active stem cells only contribute to postembryonic development of plants, apical meristems are set up and defined during embryogenesis, but remain inactive until germination. We showed previously that during stem cell activation, WUS expression is synergistically controlled by photoreceptor-mediated light signaling pathways and photosynthesis derived sugars and that both pathways require activity of the TARGET OF RAPAMYCIN (TOR) kinase (Pfeiffer et al., 2016). TOR is an evolutionary conserved regulator of growth and acts as central nutrient sensor promoting anabolic processes, like protein biosynthesis under favorable conditions, while at the same time limiting autophagy driven catabolic turnover (G. Y. Liu \& Sabatini, 2020; Shi et al., 2018; Tafur et al., 2020). By phosphorylation of central regulators of ribosome biogenesis and cell cycle regulation, such as S6K1 and E2FA, TOR controls transcriptome and metabolic reprogramming to facilitate adaptation to changes in energy- and nutrient availability (Dong et al., 2017; Xiong et al., 2013). Although much progress has been made in elucidating the TOR signaling landscape in mammals, our understanding of the TOR network in plants remains relatively poor. Recently, it has been demonstrated that TOR dependent phosphorylation of ETHYLENE INSENSITVE 2 (EIN2) controls growth of etiolated seedlings and mediates the transcriptional response to glucose in an ethylene independent manner (Fu et al., 2021). Moreover, the phytohormones auxin and abscisic acid have been identified as upstream regulators that influence TOR activity by modulating complex assembly with the associated regulatory protein RAPTOR (Li et al., 2017; P. Wang et al., 2018).

While the mechanisms connecting TOR with sugar and hormone responses have begun to emerge, little is known about how TOR controls shoot growth and SAM activity. We showed previously that TOR dependent stem cell activation correlated with increased CK signaling in the SAM and that mutations in $c k x 5$ and $c k x 6$ promoted WUS expression during germination. However, the mechanisms connecting TOR kinase and CK signaling had remained elusive. Here, we report that for controlling WUS expression and shoot development, the cytokinin $t Z$ is the most relevant downstream effector of TOR and demonstrate that translational regulation of cytokinin catabolic enzymes represents the major mechanism for TOR kinase to regulate tZ levels. 


\section{Results}

Building on our previous results that demonstrated that TOR acts as the central integrator of light and sugar signaling during stem cell activation (Pfeiffer et al., 2016), we set out to identify downstream regulatory pathways that connect TOR with WUS expression and shoot development. Therefore, we analyzed the transcriptomes of Arabidopsis shoots with impaired TOR function. Because tor null mutants are lethal (Menand et al., 2002) and Arabidopsis is insensitive to Rapamycin, we applied short term treatments with three independent TOR active site inhibitors, namely AZD8055, TORIN1 and KU63794 (Q. Liu et al., 2012; Schenone et al., 2011). We first systematically optimized working concentrations and treatment durations for all three inhibitors using S6K1 phosphorylation as a readout for TOR activity (Fig. S1). We observed robust reduction of S6K1 phosphorylation in shoots of four days old seedlings eight hours after transfer to plates containing $2 \mu \mathrm{M}$ AZD8055, $10 \mu \mathrm{M}$ TORIN1, or $20 \mu \mathrm{M}$ KU63794, with transfer to DMSO plates serving as controls. Using this setup for our RNA-seq experiments, we were able to identify the expression of 23654 genes across all samples. Using DESeq2 (Love et al., 2014), we found 6639 differentially expressed genes (DEGs) in inhibitor treated samples compared to the mock control (Fig. 1a + Table1), among which 3266 (49.2\%) showed reduced expression, whereas 3373 (50.8\%) accumulated to higher levels than in controls. Most genes were affected by AZD8055 (5303 DEGs) followed by KU63794 (4556 DEGs) and TORIN1 (3692 DEGs). The different treatments caused significantly overlapping effects, with 2509 (37.7\%) DEGs common to all three inhibitors and 4403 (66.3\%) DEGs found in at least two of three treatments, suggesting that we were able to identify a set of robust TOR sensitive transcripts. The differences between the three substances likely are caused by different pharmacokinetics and slightly different target affinities (Q. Liu et al., 2012; Schenone et al., 2011).

For further analysis we focused on mRNAs that showed a similar behavior in response to at least two inhibitors (significant difference in abundance with the same direction of change), which resulted in 4398 high confidence TOR target genes (Fig. 1a). Among the genes with reduced expression, GO categories of well characterized TOR dependent processes were most prevalent, such as translation, ribosome biogenesis, tRNA metabolism and anabolic processes (Dobrenel et al., 2016; Shi et al., 2018). The same applied to transcripts with increased accumulation among which we found GO categories related to catabolism, autophagy, secondary metabolism, glucosinolate biosynthesis and photosynthesis to be enriched (Malinovsky et al., 2017; Pu et al., 2017; Ren, 2015). Considering the largely overlapping transcriptomes in response to the three inhibitors and the high agreement of the 
gene categories with previously published data, we were confident to have obtained a high quality and highly specific dataset for early TOR response genes.

During detailed inspection of our data, we identified many DEGs related to hormone signaling pathways (Fig. 1b) with genes related to abscisic acid (ABA) but also auxin, cytokinins (CKs), jasmonic acid, ethylene, brassinosteroids and gibberellic acid being well represented (Fig. 1b). Interestingly, for most hormones the numbers of increased and reduced transcripts were similar. One notable exception was CKs, where the abundance of most transcripts was reduced in response to TOR inhibition. This apparent reduction of pathway activity caught our attention because of the known positive effect of CKs on SAM activity and shoot development (Kiba et al., 2013; Osugi et al., 2017). The transcriptome analysis revealed differential regulation of genes involved in CK biosynthesis (IPT1, -9. CYP735A2, LOG5, -8), -transport (PUP1, -14), -sensing (AHK4), -signal transmission (AHP1, -2) and -transcriptional regulation (ARR2, -10, -12) (Fig. 1c). Notably, all of the six type-A ARRs that appeared as differentially regulated in our dataset were repressed. Expression of type-A ARRs is commonly used as an approximation for CK signaling output and we thus concluded that CK signaling response is likely reduced following TOR inhibition.

To test whether these findings obtained at the level of the entire seedling shoot bear any relevance for the SAM, we used the well-established pTCSn:GUS CK signaling reporter line (Zürcher et al., 2013). In line with the RNA expression data for type-A ARRs, we found that pTCSn activity was substantially reduced after AZD8055 treatment especially in the meristematic region of the shoot, where CK function is required to maintain WUS expression (Fig. 1d). We then utilized our pWUS:3xVenus:NLS transcriptional reporter to quantify the effect of TOR inhibition on CK dependent activation of WUS. We had shown previously that WUS expression in etiolated seedlings is strongly elevated by treatment with the synthetic CK derivative 6-benzyladenin (6-BA) (Pfeiffer et al., 2016). However, this activation was almost fully blocked when 6-BA was applied together with AZD8055 (Fig. 2a). Consistently, 6-BA induced expression of several type-A ARRs was suppressed when seedlings were preincubated with the TOR inhibitor (Fig. S2). Together, this suggested that TOR inhibition interferes with the activation of various CK response genes in the SAM.

Since reduced CK signaling could be a major cause for the previously observed effects of TOR inhibition on SAM activity and WUS expression, we next aimed at identifying at which step of the pathway this effect may occur. Transcription factors of the type-B ARRs family have been shown to directly bind to the WUS promoter and induce WUS expression in response to CK sensing (Dai et al., 2017; Gruel et al., 2016; Meng et al., 2017). To test the functional interaction between TOR and type-B ARRs for WUS activation, we crossed our 
pWUS:3xVenus:NLS reporter with a line carrying an inducible version of a constitutively active allele of ARR1 ( $p 35 S: A R R 1 \triangle D D K: G R$ ). This version was expressed from a strong and ubiquitous viral promoter ( $p 35 S$ ) and the transcription factor was lacking the receiver domain $(\triangle D D K)$, making it independent of $\mathrm{CK}$ induced phosphorylation. In addition, it was fused to a glucocorticoid receptor (GR) domain, enabling dexamethasone (DEX) dependent nuclear translocation and thus experimental induction (Sakai et al., 2001b). Upon DEX treatment of p35S:ARR1 $D D K$ K:GR shoots, WUS became expressed in most cells (Fig. 2b) confirming that it is a direct CK target gene. Interestingly, treatment with AZD8055 did not interfere with this response, indicating that TOR interacts with CK signaling upstream of type-B ARRs. This was supported by the finding that ARR $1 \triangle D D K$ mediated induction of type-A $A R R$ expression also was insensitive to TOR inhibition (Fig. S3).

To further narrow down the intersection of TOR kinase activity and CK signaling, we assessed whether seedlings would still be able to respond to treatment with endogenous CKs after TOR inhibition. We therefore performed CK response assays, using trans-Zeatin ( $t Z$ ) and isopentyladenin (iP) as cytokinins, mRNA levels of ARR5 as readout, and the CK receptor double mutant cre1-2/ ahk3-7, which is insensitive to $t Z$ and $\mathrm{iP}$, as negative control (Riefler et al., 2006) (Fig. 2c). Moreover, we decided to include roots in our assay system since iP and $t Z$ are known to evoke different responses in shoots and roots.

$t Z$ application evoked a strong increase of $A R R 5$ expression compared to the cre1-2/ ahk3-7 mutant indicating a solid hormone response. However, when the seedlings were preincubated with AZD8055, hardly any difference in ARR5 expression compared with the cre12/ ahk3-7 mutant was observed, suggesting that TOR activity is required for transcriptional responses to exogenously applied $t Z$. Interestingly, treatment with the CK derivative iP had different effects. Whereas shoots seemed to be generally insensitive to iP application, wt roots showed solid ARR5 accumulation compared to the cre1-2/ ahk3-7 mutant. Notably, treatment with AZD8055 did not interfere with this response, suggesting that seedlings are in principle able to sense CKs after TOR inhibition. Since CRE1 and AHK3 CK receptors have higher affinities towards $t Z$ than iP (Romanov et al., 2006), it appeared unlikely that the differential response observed after TOR inhibition could arise at the receptor or the signal transduction level. We thus hypothesized that the functional intersection of TOR and CK must be affecting substrate availability, by either selective degradation or sequestration of specific CKs, such as tZ.

We therefore used metabolite analyses to quantify the abundance of CK molecules in response to TOR inhibition and found drastic changes in CK homeostasis following AZD8055 treatment (Fig. 3). iP active bases were enriched 2-fold, whereas the monophosphate 
precursor (iPRMP) was slightly depleted. The changes in $t Z$ derivatives were even more pronounced, as we observed a reduction of active $t Z$ bases to $50 \%$ of the mock levels after AZD8055 treatment. Moreover, a strong decrease of trans-Zeatin riboside $(t Z R)$ and the monophosphate precursor (ZZRMP) occurred in response to TOR inhibition, whereas the conjugated glycosides ( $t Z O G, t Z 7 G, t Z 9 G$ ) were almost unchanged for both $t Z$ and iP derivatives. In several studies $Z Z$ and $Z Z R$ have been shown to be the main CKs driving shoot development and WUS expression (Kasahara et al., 2004; Landrein et al., 2018; Osugi et al., 2017) and thus this drastic reduction in $t Z$ and $t Z R$ upon TOR inhibition fitted well with effects of TOR activity on WUS. Strikingly, cis-Zeatin (cZ) active bases accumulated six-fold as well as all other $c Z$ derivatives (Fig. 3). However, $c Z$ does not show relevant $C K$ activity in classical bioassays (Gajdošová et al., 2011; Kasahara et al., 2004) and in line with this it had very little effect on our WUS reporter in etiolated seedlings compared with $t Z$ and iP (Fig. S4).

Based on the striking differential accumulation of $t Z$ and $\mathrm{PP}$ after TOR inhibition and the divergent potential of these molecules to cause CK responses in the presence of AZD8055, we hypothesized that TOR controls shoot development via the regulation of $t Z$ levels. We reasoned that if reduced $t Z$ availability was causal for the reduced WUS expression observed under TOR inhibition, exogenous re-supplementation should be able to rescue WUS promoter activity. Indeed, when we supplemented AZD8055 treated seedlings with different CKs, we observed that only $t Z R$, the synthetic CK 6-BA and to a lesser extent also $t Z$ were able to rescue WUS expression under TOR inhibition (Fig. 4a). This was in agreement with previous studies, showing that $\mathrm{ZR}$ and 6-BA have stronger potential to induce WUS and shoot development compared to $t Z$ (Landrein et al., 2018; Osugi et al., 2017).

Interestingly, this behavior was different to the one observed during stem cell activation in dark grown seeldings, where 6-BA induced WUS expression was suppressed when TOR is inhibited (Fig. 2a). We therefore hypothesized that reduced availability of photoassimilates might be responsible for the different results obtained with etiolated seedlings. Indeed, when we supplied sugar together with 6-BA or $t Z$ under TOR inhibitory conditions in dark grown seedlings, WUS expression was elevated to levels even higher than with 6-BA alone (Fig. 4b, C). This result was striking, since it implied that while TOR is the central gatekeeper for light, sugar- or CK dependent activation of WUS, respectively, once sugars and CK act together, they can bypass TOR control. Noteworthy, the effect of sucrose alone varied between the experiments, but was not significantly different compared to $t Z+A Z D+S u c r o s e ~(~ p=0.2$, Students t-test). In sum, our results so far demonstrated that TOR inhibition leads to strongly reduced $t Z$ content in shoots, which in turn is causal for reduced expression of WUS and shoot growth. 
Since the relationship between TOR and WUS appeared to be mainly governed by $t Z$ availability, we next wanted to identify the mechanisms underlying differential $t Z$ accumulation. The observed reduction of $t Z$ derivatives could be the result of either reduced biosynthesis or increased turnover and our data so far supported both mechanisms. On the one hand, expression of the gene encoding the $t Z$ synthesis enzyme CYP735A2 (Takei et al., 2004) was substantially reduced following AZD8055 treatment (Fig. 1c, TableS1), suggesting biosynthesis may be causal. On the other hand, shoots showed impaired response to $t Z$ treatment upon TOR inhibition (Fig. 2c), clearly pointing towards increased $t Z$ turnover after TOR inhibition. To elucidate the relative contribution of both, we decided to investigate the role of CKX cytokinin degrading enzymes in TOR mediated control of shoot development and regulation of WUS. To this end we employed shoot fresh weight analyses as a measure of growth in response to treatment with increasing concentrations of AZD8055. These simple assays allowed us to determine ED50 values across a range of genotypes and revealed that CKX enzymes are at least partially responsible for the observed effect of TOR on cytokinin mediated growth control (Fig. 5). In contrast to wild-type seedlings, which showed growth inhibition on $0.1 \mu \mathrm{M}$ AZD8055 and were massively affected on $0.5 \mu \mathrm{M}$, seedlings with mutations in $C K X 2, C K X 3, C K X 4, C K X 5$, or $C K X 6$ were largely resistant to $0.1 \mu \mathrm{M}$ AZD8055. In addition, growth of $c k \times 4$ and $c k \times 5$ mutants, as well as $c k \times 5 / 6$ double mutants was only mildly affected on plates containing $0.5 \mu \mathrm{M}$ AZD8055, suggesting that they may represent the most relevant TOR targets. Quantitatively, however, all mutant seedlings were significantly different from wild type, which had an ED50 of $0.41 \mu \mathrm{M}$, whereas ED50 was $0.65 \mu \mathrm{M}$ for $c k x 2,1.25 \mu \mathrm{M}$ for $c k x 3$ and $0.68 \mu \mathrm{M}$ for $c k x 4$. The other CKX mutants were in the genetic background of our pCLV3/pWUS double reporter line and where therefore assayed separately. The AZD8055 ED50 value for this transgenic line was $0.64 \mu \mathrm{M}$ and again, the CKX mutants tested in the same experiment were significantly different with $0.81 \mu \mathrm{M}$ for $c k x 5,0.78 \mu \mathrm{M}$ for $c k x 6$ and 0.82 $\mu \mathrm{M}$ for $c k x 5 / c k x 6$ double mutants. Taken together, these experiments suggested that multiple CKX enzymes were likely to contribute to the effect of TOR on CK signaling. Importantly, this notion was also supported by experiments employing the general CKX inhibitor INCYDE (Zatloukal et al., 2008). Treatment with $75 \mathrm{nM}$ INCYDE made wild type seedlings fully resistant to even $0.5 \mu \mathrm{M}$ AZD8055 (Fig. $5 \mathrm{c}, \mathrm{d}$ ), demonstrating that the enhanced activity of multiple CKX enzymes is responsible for mediating the control of shoot growth by the TOR kinase.

To connect shoot growth with SAM activity and to investigate the role of $C K X$ genes in controlling WUS expression in response to TOR, we used CRISPR alleles of CKX5 and CKX6 we had generated previously in the WUS reporter background, because WUS RNA levels in 
seedlings cannot be quantified reliably by other means. Supporting our findings at the whole seedling level, we found that inactivation of $C K X 5$, or $C K X 5$ and $C K X 6$ completely abolished the negative effect of TOR inhibition on WUS expression, whereas mutation of CKX6 alone had no effect (Fig. 5b). The untreated $c k x 5,6$ double mutant exhibited higher basal expression levels of the reporter and WUS promoter activity was only reduced to wild-type levels when treated with AZD8055, demonstrating additive genetic interaction between $c k x 5$ and $c k x 6$ independent of TOR input. Again, as for shoot growth, general pharmacological inhibition of CKX activity using INCYDE (Zatloukal et al., 2008) also rescued WUS expression to mock levels in the presence of $2 \mu \mathrm{M}$ AZD8055 (Fig. 5d). Together, these observations demonstrated that increased CKX activity upon TOR inhibition caused reduced WUS expression and in turn impaired shoot growth, pointing towards a molecular interaction between TOR and the CKX enzyme family.

TOR could affect CKX enzymes at various levels from expression of the corresponding genes, to CKX mRNA translation, to enzyme activity, or CKX protein stability. Mining our transcriptome data, we found no evidence of differential regulation of $C K X$ isoforms at the transcript level, which we were able to confirm by RT-qPCR (Fig. S5). Only CKX6 showed a mild but significant increase in transcript abundance, whereas $C K X 2$ and $C K X 5$ transcripts were even decreased. Hence, we concluded that the nature of the molecular interaction between TOR and CKXs likely occurs downstream of the mRNA level. To distinguish between increased protein accumulation and CKX enzyme activity, we analyzed protein abundance in response to AZD8055. Since there are currently no antibodies against any of the CKX enzymes available, we made use of a p35S:cMyc-CKX1 translational fusion line (Niemann et al., 2015, 2018). Western blot analysis revealed that eight hours after transfer to AZD8055 containing medium, seedlings accumulated 39 - $109 \%$ more cMyc-CKX1 than mock treated seedlings in three independent experiments (Fig. 6a). In contrast, AZD8055 did not induce significant changes in CKX1 transcript abundance in the investigated line (Fig. 6b) in the experiment. Protein accumulation is either the result of decreased protein turnover or increased protein biosynthesis. We first tested if CKX protein stability was affected by TOR inhibition. To this end we utilized cycloheximide to inhibit translational elongation and assayed cMyc-CKX1 protein levels over the course of eight hours with or without preincubation on AZD8055. We found that in AZD8055 and mock treated seedlings the turnover rate was very similar and the protein half-life was determined at $\sim 4 \mathrm{~h}$ for both, which is in agreement with published results (Niemann et al., 2015). As a result, we concluded that TOR inhibition does not affect $\mathrm{CKX} 1$ protein stability but may rather enhance CKX1 translation to cause the observed protein over-accumulation (Fig. 6a). To test this directly, we applied polysome 
profiling to analyze whether TOR inhibition modulates the association of different $C K X \mathrm{mRNAs}$ with highly translating polysomes (Fig. $7 a+S 6+S 7$ ). To this end, we prepared polysome fractions from mock and AZD8055 treated seedlings using sucrose gradient centrifugation and quantified the abundance of $C K X$ mRNAs in the fractions using RT-qPCR. Consistent with the enhanced protein accumulation of CKX1, we observed a strong enrichment of $C K X 1 \mathrm{mRNA}$ in the heavy polysome fractions (fractions 1-3), which suggested that more CKX1 protein was synthesized at the ribosomes after eight hours of TOR inhibition compared to mock treated controls. Similarly, we observed accumulation of CKX5 transcripts in polysomal fractions (Fig. 7b), which fitted well with the observed resistance of the ckx5 mutant towards AZD8055 treatment (Fig. $5 a+5 b)$. We also observed subtle accumulation of $C K X 6$ and $C K X 7$ transcripts in some of the lighter polysomal fractions (fractions 3-5) following AZD8055 treatment (Fig. 7b + S7). The lower enrichment of $C K X 6$ mRNAs in polysomes compared to $C K X 5$ fitted well with the behavior in our physiological assays observed for the respective mutants (Fig. $5 \mathrm{a}+\mathrm{Fig}$. $5 b)$. In contrast, CKX4 mRNA appeared rather depleted from polysomes following TOR inhibition, although this effect was not consistently observed in all experimental repetitions (Fig. 7b). In conclusion, our experiments showed that translation of multiple transcripts encoding CKX enzymes is controlled by TOR activity. In particular, CKX1 and CKX5 protein biosynthesis appeared to be repressed when TOR is active, which fitted well with functional data on CKX5. In contrast, translation of CKX4 appeared unaffected or even enhanced by TOR activity, whereas our genetic data showed a clear antagonistic effect of both players (Fig. $5 e)$. Close inspection of the $5^{\prime}$-leader sequences of all $C K X$ encoding genes revealed that only CKX4 contains a strong uORF signature that may influence the observed response to TOR inhibition.

In sum, our results demonstrated that the TOR kinase controls WUS expression and shoot development via regulation of cytokinin levels, specifically $t Z$. This effect is mediated through modulation of protein accumulation of specific CKX cytokinin degrading enzymes. Mechanistically, TOR controls CKX protein accumulation at the transcriptional level, but more importantly, by repressing CKX mRNA translation.

\section{Discussion}

Plants dynamically adjust their transcriptional and translational programs to adapt to diverse environments. The TOR kinase network is a master regulator of cellular metabolism and controls translation in accordance with environmental parameters in all eukaryotes. Several reports have shown a connection between TOR activity and stem cell regulation and identified 
numerous upstream regulators of the TOR complex and stem cell activity (Li et al., 2017; Pfeiffer et al., 2016; Xiong et al., 2013). However, the downstream effectors mediating TOR effects on stem cells of the shoot apical meristem so far remained elusive. In this study, we show that TOR controls translation of individual CKX isoforms (Fig. 7), resulting in increased CKX protein levels (Fig. 6a). CKX activity in turn limits the availability of trans-Zeatin, the major instructive signal for SAM activity and WUS expression (Fig. 3 + Fig. 4a).

CKX enzymes catalyze the irreversible degradation of CKs and hence exert strong influence on development and growth (Bartrina et al., 2011). Cytokinins are potent regulators of plant growth and development and act as long-distance signaling molecules that instruct cellular identity and behavior of the SAM (Kieber \& Schaller, 2014; Landrein et al., 2018; Osugi et al., 2017). Thus, controlling CK abundance is key to synchronize the SAM with cellular homeostasis of distant source tissues that acquire the resources necessary for further organogenesis. Our results suggest that TOR under favorable conditions represses translation of CKX mRNAs, whereas when TOR is inhibited CKX translational efficiency is enhanced resulting in globally reduced levels of trans-Zeatin and ultimately in reduced activity of stem cells in the SAM. This active translational repression enables global adjustments of CK availability withing minutes and allows the plant to respond to environmental changes much faster than by relying on a transcriptional response alone. Our results so far do not allow conclusions about the detailed molecular mechanism by which TOR activity results in translational repression of specific transcripts. One potential mechanism might involve SnRK1 dependent translation mediated by eiFiso4G, although in published translational efficiency data of eiFiso4G mutants no evidence for differential translation of CKX isoforms was found (Cho et al., 2019). Also GCN2 dependent translation via eif2alpha is a potential pathway that could drive translation antagonistically to TOR (Sesma et al., 2017). Our polysome data shows very specific responses of CKX isoforms in seedling shoots. In addition to transcript specificity, we speculate that differential regulatory responses may also exist in different cellular contexts. Cell type specific TOR functions have also been suggested by a recent study showing differences in TOR activity between source and sink tissues resulting in inverse regulation of plasmodesmata permeability (Brunkard et al., 2020).

Interfering with CK homeostasis through targeted expression of CKXs can increase plants resilience to environmental stresses such as drought or cold and improve yield traits (Ashikari et al., 2005; Cortleven et al., 2019; Nehnevajova et al., 2019; Schwarz et al., 2020) and are thus perceived as promising candidates of agronomic importance (Jameson \& Song, 2020). Consequently, misexpression of CKXs has been successfully employed to generate improved crops and targeted translational control bears the potential to generate cultivars that 
ameliorate the detrimental effects of broad CKX overexpression. Along these lines, we observed that the supplementation of sugars together with CKs is sufficient to drive WUS expression even when TOR is inactive. This further fuels the question about specific TOR functions in stem cells compared to differentiated tissues, since in this context CKs are not degraded in the SAM, although some CKX isoforms are specifically expressed in the OC (Bartrina et al., 2011).

Our findings on TOR mediated translational repression of catabolic enzymes open new avenues to study the mechanisms of TOR driven environmental adaptation. Considering the ecological benefits that could conceptually be derived from tunable translational repression of growth factor catabolism, this regulatory logic may be much more widespread than anticipated so far. Thus, future studies might reveal analogous mechanisms for other catabolic genes in plants or animal systems. 


\section{Materials and methods}

\section{Plant material}

All used plant lines were in the Col-0 background. The double reporter line pWUS:3xVenus:NLS/ pCLV3:mCherry:NLS as well as the $c k x 5, c k x 6$ and $c k x 5 / c k x 6$ CRISPR mutants are described in (Pfeiffer et al., 2016). The p35S:ARR1 $\triangle D D K$ :GR line was described in (Sakai et al., 2001a) and was crossed with the double reporter line to obtain homozygous alleles for each transgene. The p35S:cMyc-CKX1 line is described in (Niemann et al., 2015, 2018). The ckx2 (SALK_083761c), ckx3 (SALK_050938c), ckx4 (SALK_055204c) mutants were obtained from NASC. The cre1-2/ahk3-7 mutant was described in (Riefler et al., 2006).

\section{Growth conditions}

Seeds were sterilized with $70 \%$ ethanol and $0.1 \%$ Triton for $10 \mathrm{~min}$ and afterwards washed twice with autoclaved water. Seeds were plated on $100 \mu$ m nylon meshes (nitex 03/100-44, Sefar, Heiden, Switzerland) on top of $0.5 x$ MS (Duchefa, Haarlem, The Netherlands), $0.9 \%$ Phytoagar in square petri dishes. After plating, seeds were imbibed for 3 days at $4^{\circ} \mathrm{C}$ in darkness and transferred to growth cabinets (poly klima, PK 520-LED, Freising, Germany) where they were kept under continuous light at $22^{\circ} \mathrm{C}$ and grown vertically for 4 days. Subsequently, seedlings were transferred with the nylon meshes to $0.5 \mathrm{x}$ MS plates supplemented with $2 \mu \mathrm{M}$ AZD8055 (Selleckchem, Houston, TX) or equal volumes of DMSO for $8 \mathrm{~h}$.

\section{Liquid culture}

About 30-40 seeds, that were imbibed as described above, were sown in $3 \mathrm{ml} 0.5 \mathrm{x}$ MS in petri dishes of $35 \mathrm{~mm}$ diameter. Plants were kept in darkness for three days after the induction of germination by $6 \mathrm{hr}$ light treatment. The medium of two day old etiolated seedlings was supplemented with the indicated treatments. All stock solutions were $1000 x$ concentrated and diluted in DMSO, therefore control plants were mock treated with the same volume of DMSO.

\section{RNAseq}

Seedlings were grown as described under growth conditions. 4 days after germination seedlings were transferred on a mesh to $0.5 \mathrm{xMS}$ plates containing either $2 \mu \mathrm{M}$ AZD8055, 10 $\mu \mathrm{M}$ TORIN1, $20 \mu \mathrm{M}$ KU63794 or equal volumes of DMSO as mock control. After $8 \mathrm{~h}, 30 \mathrm{mg}$ of shoot tissue were harvested for each replicate and frozen in liquid nitrogen. 3 independent replicates were harvested for each condition. Total RNA was extracted with the Plant RNA 
Purification Reagent (Invitrogen, Carlsbad, CA) according to the instructions of the manufacturer, digested with TURBO DNAse (Ambion/ Thermo Fisher, Waltham, MA) and purified with RNeasy Mini Kit (Quiagen, Hilden, Germany). Libraries were poly-(A) selected and analyzed with NEXTseq 500. For differential gene expression analysis reads were aligned with RNA STAR (v2.6) alignment tool with TAIR10 genome model as reference. Transcripts were assembled and counted with StringTie (v1.3.3) and statistical analysis was performed using DESeq2 (v1.18.1) (Love et al., 2014). GO term analysis was performed using ThaleMine web tool (https://www.bar.utoronto.ca/thalemine/begin.do).

Histochemical GUS staining

Four day old seedlings were harvested in $90 \%$ acetone and incubated at $-20^{\circ} \mathrm{C}$ for at least $1 \mathrm{hr}$. Seedlings were washed with PBS and incubated in substrate buffer (1x PBS (pH 7.0), $1 \mathrm{mM} \mathrm{K}_{3} \mathrm{Fe}(\mathrm{III})(\mathrm{CN})_{6}, 0.5 \mathrm{mM} \mathrm{K}_{4} \mathrm{Fe}(\mathrm{II})(\mathrm{CN})_{6}, 1 \mathrm{mM}$ EDTA, $1 \%$ Triton X-100, $1 \mathrm{mg} / \mathrm{ml} \mathrm{X-gluc}$ ) at $22^{\circ} \mathrm{C}$ over night. After staining, the seedlings were incubated with $60 \%$ and subsequently in $95 \%$ ethanol to remove chlorophyll.

Microscopy and fluorescence quantification

All images were obtained using Zeiss Imager M1, the Plan-APOCHROMAT 20x/0.8 objective (Zeiss, Oberkochen, Germany) and YFP- and GFP-specific filter sets. Procedures for fluorescent reporter activities of the double reporter were performed as described in (Pfeiffer et al., 2016). Each measurement was normalized to the median (set to 100) of the mock for experiments in the light or to the 6-BA treated samples for experiments performed in the dark.

\section{Western blot}

Approximately $30 \mathrm{mg}$ of shoot tissue were harvested, and proteins were extracted with 1:4 ratio $(\mathrm{mg} / \mu \mathrm{l})$ adjusted to the exact fresh weight with $95^{\circ} \mathrm{C}$ hot denaturing buffer (100 mM MOPS $\mathrm{pH}$ 7.6, $100 \mathrm{mM} \mathrm{NaCl}, 40 \mathrm{mM}$ B-MeOH, 5\% SDS, 10\% Glycerol, 4 mM EDTA, 2 mM PMSF, $\mathrm{PI}$ (Sigma)) and boiled at $95^{\circ} \mathrm{C}$ for $5 \mathrm{~min}$. Cellular debris was removed by two centrifugation steps (10 min, 14,000 rpm, RT). Equal volumes of the obtained extract were separated on a $10 \%$ SDS-PAGE gel and blotted to a PVDF membrane. Membranes were probed with Phospho-p70 S6 kinase (Thr(P)-389) polyclonal antibody (No.9205, Cell Signaling Technology, Cambridge, UK) to detect S6K phosphorylation. S6K1/2 antibody (AS12-1855, Agrisera AB, Vännäs, Sweden) was used to detect total S6K1 and S6K2. c-Myc antibody (9E10, Santa Cruz Biotechnology, Dallas, TX) was used to detect C-Myc tagged CKX1. 
Physiology

Seeds were singled out on $0.5 \times \mathrm{MS}, 0.9 \%$ Phytoagar plates and imbibed for three days at $4^{\circ} \mathrm{C}$ in the dark. Plates were kept horizontally in long day conditions at $22^{\circ} \mathrm{C}$ for four days. $\sim 40$ single seedlings at the same developmental stage and of similar size were selected and transferred to plates containing the indicated AZD8055 concentrations and grown for seven more days before shoot fresh weight was measured. For the measurements, seedling shoots were removed and weighed in batches of $5-10$ seedlings. Afterwards the average weight was calculated for each batch. Mixed ANOVA statistical analysis was performed in R (v4.0.2) with pooled data from two experiments. ED50 values were calculated using the "drc" package in R (Ritz C, Strebig JC, 2016)

\section{$\mathrm{CHX}$ chase assay}

P35S:CMyc-CKX1 seedlings were grown as described under growth conditions. $8 \mathrm{~h}$ after transfer to $2 \mu \mathrm{M}$ AZD8055 the plates were flooded with $50 \mathrm{ml} 200 \mu \mathrm{M}$ cycloheximide and $2 \mu \mathrm{M}$ AZD8055 solution $(0.015 \%$ Silwet L-77) for $0,2,4$ and $8 \mathrm{~h}$ and shoots were harvested for western blot analysis as described above.

\section{RT-qPCR}

Total RNA was extracted as described under RNAseq. RNA integrity was confirmed on an agarose gel and the concentrations were determined with a nanodrop device. Equal amounts of RNA were used for oligo dT primed cDNA synthesis with the RevertAid First Strand cDNA Synthesis Kit (Thermo Fisher, Waltham, MA). The qPCR reaction was set up using the SG qPCR Master Mix (EURx, Gdansk, Poland) and run on a qTOWER ${ }^{3}$ (Analytic Jena, Jena, Germany) PCR System with technical duplicates each.

\section{Cytokinin profiling - LC-MS}

For cytokinin profiling seedlings were grown as described for RNAseq analysis and 5 biological replicates of shoot tissue were harvested for each condition. The CK content was determined by ultra-high performance liquid chromatography-electrospray tandem mass spectrometry (Svačinová et al., 2012), including modifications described by (Antoniadi et al., 2015). Briefly, samples (20 mg FW) were homogenized and extracted in $1 \mathrm{ml}$ of modified Bieleski buffer $(60 \%$ methanol, $10 \% \mathrm{HCOOH}$ and $30 \% \mathrm{H}_{2} \mathrm{O}$ ) together with a cocktail of stable isotope-labeled internal standards $(0.25 \mathrm{pmol}$ of $\mathrm{CK}$ bases, ribosides, $\mathrm{N}$-glucosides, and $0.5 \mathrm{pmol}$ of $\mathrm{CK} O$ glucosides, nucleotides per sample added). The extracts were purified onto an Oasis MCX column (30 mg/1 ml, Waters) and then analyzed using using an Acquity I-class system 
(Waters, Milford, MA, USA) combined with a mass spectrometer Xevo ${ }^{\mathrm{TM}} \mathrm{TQ}-\mathrm{XS}$ (Waters, Manchester, UK). Data were processed with Target Lynx V4.2 software and final concentration levels of phytohormones were calculated using isotope dilution method (Novák et al., 2008).

Polysome fractionation

$200 \mathrm{mg}$ plant material grown as described under growth conditions was homogenized by rotating at $4^{\circ} \mathrm{C}$ in 600 ul polysome extraction buffer $(0.2 \mathrm{mM}$ Tris-HCL, $\mathrm{pH}=9,0.2 \mathrm{mM} \mathrm{KCL}, 25$ mM EGTA, 35 mM MgCl2, 1\% DOC, 1\% PTE, 1\% Brij-35, 1\% Triton X-100, 1\% NP-40, 1\% Tween-20, $5 \mathrm{mM}$ DTT, $10 \mu \mathrm{M}$ MG-132, $50 \mu \mathrm{g} / \mathrm{ml}$ Cycloheximide, $50 \mu \mathrm{g} / \mathrm{ml}$ chloramphenicol and $1 \%$ EDTA-free protease inhibitor cocktail). Extracts were centrifuged at $16000 \mathrm{xg}$ at $4{ }^{\circ} \mathrm{C}$ for $10 \mathrm{~min}$. $300 \mu \mathrm{l}$ supernatant was loaded to $7-47 \%$ sucrose gradient and centrifuged at $38000 x$ rpm for 3 hours in a Beckmann SW41Ti rotor. The gradient was fractionated after recording the absorbance at $254 \mathrm{~nm}$. RNA was precipitated from $1 \mathrm{ml}$ fraction by mix and incubation with one volume of $8 \mathrm{M}$ Guanidine-HCL and two columns of absolute ethanol at $20^{\circ} \mathrm{C}$ over night followed by centrifuge at max. speed for 1 hour. RNA pellet was resuspended with $50 \mu$ l DEPC water. 100 ng RNA was used for cDNA synthesis (SuperScript IV reverse transcriptase (ThermoFisher, 18090050).

Cytokinin response assay

Seedlings were grown as described under growth conditions. After $8 \mathrm{~h}$ of AZD8055 or mock treatment seedlings were sprayed with an atomizer with either $100 \mathrm{nM}$ of trans-Zeatin (Duchefa, Haarlem, The Netherlands) or $100 \mathrm{nM}$ of isopentyladenine (Duchefa, Haarlem, The Netherlands) solution (0.015\% Silwet L-77). After 30 min three independent replicates of shoots and roots were harvested separately for total RNA extraction and RT-qPCR analysis as described above. 


\section{References}

Antoniadi, I., Novák, O., Gelová, Z., Johnson, A., Plíhal, O., Simerský, R., Mik, V., Vain, T., Mateo-Bonmatí, E., Karady, M., Pernisová, M., Plačková, L., Opassathian, K., Hejátko, J., Robert, S., Friml, J., Doležal, K., Ljung, K., \& Turnbull, C. (2020). Cellsurface receptors enable perception of extracellular cytokinins. Nature Communications, 11(1), 4284. https://doi.org/10.1038/s41467-020-17700-9

Antoniadi, I., Plačková, L., Simonovik, B., Doležal, K., Turnbull, C., Ljung, K., \& Novák, O. (2015). Cell-type-specific cytokinin distribution within the Arabidopsis primary root apex. The Plant Cell, 27(7), 1955-1967.

Ashikari, M., Sakakibara, H., Lin, S., Yamamoto, T., Takashi, T., Nishimura, A., Angeles, E. R., Qian, Q., Kitano, H., \& Matsuoka, M. (2005). Cytokinin Oxidase Regulates Rice Grain Production. Science, 309(5735), 741-745. https://doi.org/10.1126/science.1113373

Bartrina, I., Otto, E., Strnad, M., Werner, T., \& Schmülling, T. (2011). Cytokinin regulates the activity of reproductive meristems, flower organ size, ovule formation, and thus seed yield in Arabidopsis thaliana. The Plant Cell, 23(1), 69-80.

Bäurle, I., \& Laux, T. (2005). Regulation of WUSCHEL transcription in the stem cell niche of the Arabidopsis shoot meristem. The Plant Cell, 17(8), 2271-2280.

Brunkard, J. O., Xu, M., Scarpin, M. R., Chatterjee, S., Shemyakina, E. A., Goodman, H. M., \& Zambryski, P. (2020). TOR dynamically regulates plant cell-cell transport.

Proceedings of the National Academy of Sciences, 117(9), 5049-5058. https://doi.org/10.1073/pnas.1919196117

Buechel, S., Leibfried, A., To, J. P. C., Zhao, Z., Andersen, S. U., Kieber, J. J., \& Lohmann, J. U. (2010). Role of A-type ARABIDOPSIS RESPONSE REGULATORS in meristem maintenance and regeneration. European Journal of Cell Biology, 89(2), 279-284. https://doi.org/10.1016/j.ejcb.2009.11.016

Cho, H.-Y., Lu, M.-Y. J., \& Shih, M.-C. (2019). The SnRK1-elFiso4G1 signaling relay regulates the translation of specific mRNAs in Arabidopsis under submergence. New Phytologist, 222(1), 366-381. https://doi.org/10.1111/nph.15589

Cortleven, A., Leuendorf, J. E., Frank, M., Pezzetta, D., Bolt, S., \& Schmülling, T. (2019). Cytokinin action in response to abiotic and biotic stresses in plants. Plant, Cell \& Environment, 42(3), 998-1018. https://doi.org/10.1111/pce.13494

Dai, X., Liu, Z., Qiao, M., Li, J., Li, S., \& Xiang, F. (2017). ARR12 promotes de novo shoot regeneration in Arabidopsis thaliana via activation of WUSCHEL expression. 59(10), 12.

Daum, G., Medzihradszky, A., Suzaki, T., \& Lohmann, J. U. (2014). A mechanistic framework for noncell autonomous stem cell induction in Arabidopsis. Proceedings of the National Academy of Sciences, 111(40), 14619-14624.

Dobrenel, T., Caldana, C., Hanson, J., Robaglia, C., Vincentz, M., Veit, B., \& Meyer, C. (2016). TOR Signaling and Nutrient Sensing. Annual Review of Plant Biology, 67(1), 261-285. https://doi.org/10.1146/annurev-arplant-043014-114648

Dong, Y., Silbermann, M., Speiser, A., Forieri, I., Linster, E., Poschet, G., Allboje Samami, A., Wanatabe, M., Sticht, C., Teleman, A. A., Deragon, J.-M., Saito, K., Hell, R., \& Wirtz, M. (2017). Sulfur availability regulates plant growth via glucose-TOR signaling. Nature Communications, 8(1), 1174. https://doi.org/10.1038/s41467-017-01224-w 
Fu, L., Liu, Y., Qin, G., Wu, P., Zi, H., Xu, Z., Zhao, X., Wang, Y., Li, Y., Yang, S., Peng, C., Wong, C. C. L., Yoo, S.-D., Zuo, Z., Liu, R., Cho, Y.-H., \& Xiong, Y. (2021). The TOR-EIN2 axis mediates nuclear signalling to modulate plant growth. Nature, 591(7849), 288-292. https://doi.org/10.1038/s41586-021-03310-y

Gajdošová, S., Spíchal, L., Kamínek, M., Hoyerová, K., Novák, O., Dobrev, P. I., Galuszka, P., Klíma, P., Gaudinová, A., Žižková, E., Hanuš, J., Dančák, M., Trávníček, B., Pešek, B., Krupička, M., Vaňková, R., Strnad, M., \& Motyka, V. (2011). Distribution, biological activities, metabolism, and the conceivable function of cis-zeatin-type cytokinins in plants. Journal of Experimental Botany, 62(8), 2827-2840.

https://doi.org/10.1093/jxb/erq457

Gordon, S. P., Chickarmane, V. S., Ohno, C., \& Meyerowitz, E. M. (2009). Multiple feedback loops through cytokinin signaling control stem cell number within the Arabidopsis shoot meristem. Proceedings of the National Academy of Sciences, 106(38), 1652916534. https://doi.org/10.1073/pnas.0908122106

Gruel, J., Landrein, B., Tarr, P., Schuster, C., Refahi, Y., Sampathkumar, A., Hamant, O., Meyerowitz, E. M., \& Jönsson, H. (2016). An epidermis-driven mechanism positions and scales stem cell niches in plants. Science Advances, 2(1), e1500989. https://doi.org/10.1126/sciadv.1500989

Heyl, A., Werner, T., \& Schmülling, T. (2018). Cytokinin Metabolism and Signal Transduction. In J. A. Roberts (Ed.), Annual Plant Reviews online (pp. 93-123). John Wiley \& Sons, Ltd. https://doi.org/10.1002/9781119312994.apr0245

Holst, K., Schmülling, T., \& Werner, T. (2011). Enhanced cytokinin degradation in leaf primordia of transgenic Arabidopsis plants reduces leaf size and shoot organ primordia formation. Journal of Plant Physiology, 168(12), 1328-1334.

Jameson, P. E., \& Song, J. (2020). Will cytokinins underpin the second 'Green Revolution'? Journal of Experimental Botany, 71(22), 6872-6875. https://doi.org/10.1093/jxb/eraa447

Janocha, D., \& Lohmann, J. U. (2018). From signals to stem cells and back again. Current Opinion in Plant Biology, 45, 136-142. https://doi.org/10.1016/j.pbi.2018.06.005

Kasahara, H., Takei, K., Ueda, N., Hishiyama, S., Yamaya, T., Kamiya, Y., Yamaguchi, S., \& Sakakibara, H. (2004). Distinct Isoprenoid Origins of cis- and trans-Zeatin Biosyntheses in Arabidopsis. Journal of Biological Chemistry, 279(14), 14049-14054. https://doi.org/10.1074/jbc.M314195200

Kiba, T., Takei, K., Kojima, M., \& Sakakibara, H. (2013). Side-Chain Modification of Cytokinins Controls Shoot Growth in Arabidopsis. Developmental Cell, 27(4), 452461. https://doi.org/10.1016/j.devcel.2013.10.004

Kieber, J. J., \& Schaller, G. E. (2014). Cytokinins. The Arabidopsis Book / American Society of Plant Biologists, 12. https://doi.org/10.1199/tab.0168

Köllmer, I., Novák, O., Strnad, M., Schmülling, T., \& Werner, T. (2014). Overexpression of the cytosolic cytokinin oxidase/dehydrogenase (CKX 7) from A rabidopsis causes specific changes in root growth and xylem differentiation. The Plant Journal, 78(3), 359-371.

Kuroha, T., Tokunaga, H., Kojima, M., Ueda, N., Ishida, T., Nagawa, S., Fukuda, H., Sugimoto, K., \& Sakakibara, H. (2009). Functional Analyses of LONELY GUY Cytokinin-Activating Enzymes Reveal the Importance of the Direct Activation Pathway in Arabidopsis. The Plant Cell, 21(10), 3152-3169. https://doi.org/10.1105/tpc.109.068676 
Landrein, B., Formosa-Jordan, P., Malivert, A., Schuster, C., Melnyk, C. W., Yang, W., Turnbull, C., Meyerowitz, E. M., Locke, J. C. W., \& Jönsson, H. (2018). Nitrate modulates stem cell dynamics in Arabidopsis shoot meristems through cytokinins. Proceedings of the National Academy of Sciences, 115(6), 1382-1387. https://doi.org/10.1073/pnas.1718670115

Laux, T., Mayer, K. F. X., Berger, J., \& Jürgens, G. (1996). The WUSCHEL gene is required for shoot and floral meristem integrity in Arabidopsis. 10.

Li, X., Cai, W., Liu, Y., Li, H., Fu, L., Liu, Z., Xu, L., Liu, H., Xu, T., \& Xiong, Y. (2017). Differential TOR activation and cell proliferation in Arabidopsis root and shoot apexes. Proceedings of the National Academy of Sciences, 114(10), 2765-2770. https://doi.org/10.1073/pnas.1618782114

Liu, G. Y., \& Sabatini, D. M. (2020). MTOR at the nexus of nutrition, growth, ageing and disease. Nature Reviews Molecular Cell Biology, 21(4), 183-203. https://doi.org/10.1038/s41580-019-0199-y

Liu, Q., Kirubakaran, S., Hur, W., Niepel, M., Westover, K., Thoreen, C. C., Wang, J., Ni, J., Patricelli, M. P., Vogel, K., Riddle, S., Waller, D. L., Traynor, R., Sanda, T., Zhao, Z., Kang, S. A., Zhao, J., Look, A. T., Sorger, P. K., ... Gray, N. S. (2012). Kinome-wide Selectivity Profiling of ATP-competitive Mammalian Target of Rapamycin (mTOR) Inhibitors and Characterization of Their Binding Kinetics ${ }^{*}$, . Journal of Biological Chemistry, 287(13), 9742-9752. https://doi.org/10.1074/jbc.M111.304485

Love, M. I., Huber, W., \& Anders, S. (2014). Moderated estimation of fold change and dispersion for RNA-seq data with DESeq2. Genome Biology, 15(12), 550. https://doi.org/10.1186/s13059-014-0550-8

Malinovsky, F. G., Thomsen, M.-L. F., Nintemann, S. J., Jagd, L. M., Bourgine, B., Burow, M., \& Kliebenstein, D. J. (2017). An evolutionarily young defense metabolite influences the root growth of plants via the ancient TOR signaling pathway. ELife, 6, e29353. https://doi.org/10.7554/eLife.29353

Mayer, K. F. X., Schoof, H., Haecker, A., Lenhard, M., Jürgens, G., \& Laux, T. (1998). Role of WUSCHEL in Regulating Stem Cell Fate in the Arabidopsis Shoot Meristem. Cell, 95(6), 805-815. https://doi.org/10.1016/S0092-8674(00)81703-1

Menand, B., Desnos, T., Nussaume, L., Berger, F., Bouchez, D., Meyer, C., \& Robaglia, C. (2002). Expression and disruption of the Arabidopsis TOR (target of rapamycin) gene. Proceedings of the National Academy of Sciences, 99(9), 6422-6427. https://doi.org/10.1073/pnas.092141899

Meng, W. J., Cheng, Z. J., Sang, Y. L., Zhang, M. M., Rong, X. F., Wang, Z. W., Tang, Y. Y., \& Zhang, X. S. (2017). Type-B ARABIDOPSIS RESPONSE REGULATORs Specify the Shoot Stem Cell Niche by Dual Regulation of WUSCHEL. The Plant Cell, 29(6), 1357-1372. https://doi.org/10.1105/tpc.16.00640

Nehnevajova, E., Ramireddy, E., Stolz, A., Gerdemann-Knörck, M., Novák, O., Strnad, M., \& Schmülling, T. (2019). Root enhancement in cytokinin-deficient oilseed rape causes leaf mineral enrichment, increases the chlorophyll concentration under nutrient limitation and enhances the phytoremediation capacity. BMC Plant Biology, 19(1), 83. https://doi.org/10.1186/s12870-019-1657-6

Niemann, M. C. E., Bartrina, I., Ashikov, A., Weber, H., Strnad, M., Strasser, R., Bakker, H., \& Schmülling, T. (2015). Arabidopsis ROCK1 transports UDP-GlcNAc/UDP-GalNAc and regulates ER protein quality control and cytokinin activity. PLANT BIOLOGY, 6. 
Niemann, M. C. E., Weber, H., Hluska, T., Leonte, G., Anderson, S. M., Novák, O., Senes, A., \& Werner, T. (2018). The Cytokinin Oxidase/Dehydrogenase CKX1 Is a Membrane-Bound Protein Requiring Homooligomerization in the Endoplasmic Reticulum for Its Cellular Activity. Plant Physiology, 176(3), 2024-2039. https://doi.org/10.1104/pp.17.00925

Novák, O., Hauserová, E., Amakorová, P., Doležal, K., \& Strnad, M. (2008). Cytokinin profiling in plant tissues using ultra-performance liquid chromatography-electrospray tandem mass spectrometry. Phytochemistry, 69(11), 2214-2224.

https://doi.org/10.1016/j.phytochem.2008.04.022

Osugi, A., Kojima, M., Takebayashi, Y., Ueda, N., Kiba, T., \& Sakakibara, H. (2017). Systemic transport of trans-zeatin and its precursor have differing roles in Arabidopsis shoots. Nature Plants, 3(8), 17112. https://doi.org/10.1038/nplants.2017.112

Pfeiffer, A., Janocha, D., Dong, Y., Medzihradszky, A., Schöne, S., Daum, G., Suzaki, T., Forner, J., Langenecker, T., Rempel, E., Schmid, M., Wirtz, M., Hell, R., \& Lohmann, J. U. (2016). Integration of light and metabolic signals for stem cell activation at the shoot apical meristem. ELife, 5, e17023. https://doi.org/10.7554/eLife.17023

Pu, Y., Luo, X., \& Bassham, D. C. (2017). TOR-Dependent and -Independent Pathways Regulate Autophagy in Arabidopsis thaliana. Frontiers in Plant Science, 8, 1204. https://doi.org/10.3389/fpls.2017.01204

Ren, M. (2015). TOR-inhibitor insensitive-1 (TRIN1) regulates cotyledons greening in Arabidopsis. Frontiers in Plant Science, 6, 13.

Riefler, M., Novak, O., Strnad, M., \& Schmülling, T. (2006). Arabidopsis Cytokinin Receptor Mutants Reveal Functions in Shoot Growth, Leaf Senescence, Seed Size, Germination, Root Development, and Cytokinin Metabolism. The Plant Cell, 18(1), 40-54. https://doi.org/10.1105/tpc.105.037796

Ritz C, Strebig JC. (2016). Package 'drc'. https://www.r-project.org/

Romanov, G. A., Lomin, S. N., \& Schmülling, T. (2006). Biochemical characteristics and ligand-binding properties of Arabidopsis cytokinin receptor AHK3 compared to CRE1/AHK4 as revealed by a direct binding assay. Journal of Experimental Botany, 57(15), 4051-4058. https://doi.org/10.1093/jxb/erl179

Sakai, H., Honma, T., Aoyama, T., Sato, S., Kato, T., Tabata, S., \& Oka, A. (2001b). ARR1, a Transcription Factor for Genes Immediately Responsive to Cytokinins. Science, 294(5546), 1519-1521. https://doi.org/10.1126/science.1065201

Schenone, S., C., B., F., M., M., R., \& M., B. (2011, June 30). ATP-Competitive Inhibitors of mTOR: An Update. Current Medicinal Chemistry. https://www.eurekaselect.com/74340/article

Schoof, H., Lenhard, M., Haecker, A., Mayer, K. F. X., Jürgens, G., \& Laux, T. (2000). The Stem Cell Population of Arabidopsis Shoot Meristems Is Maintained by a Regulatory Loop between the CLAVATA and WUSCHEL Genes. Cell, 100(6), 635-644. https://doi.org/10.1016/S0092-8674(00)80700-X

Schwarz, I., Scheirlinck, M.-T., Otto, E., Bartrina, I., Schmidt, R.-C., \& Schmülling, T. (2020). Cytokinin regulates the activity of the inflorescence meristem and components of seed yield in oilseed rape. Journal of Experimental Botany, 71(22), 7146-7159. https://doi.org/10.1093/jxb/eraa419 
Sesma, A., Castresana, C., \& Castellano, M. M. (2017). Regulation of Translation by TOR, elF4E and elF2a in Plants: Current Knowledge, Challenges and Future Perspectives. Frontiers in Plant Science, 8. https://doi.org/10.3389/fpls.2017.00644

Shi, L., Wu, Y., \& Sheen, J. (2018). TOR signaling in plants: Conservation and innovation. Development, 145(13).

Svačinová, J., Novák, O., Plačková, L., Lenobel, R., Holík, J., Strnad, M., \& Doležal, K. (2012). A new approach for cytokinin isolation from Arabidopsis tissues using miniaturized purification: Pipette tip solid-phase extraction. Plant Methods, 8(1), 17. https://doi.org/10.1186/1746-4811-8-17

Tafur, L., Kefauver, J., \& Loewith, R. (2020). Structural insights into TOR signaling. Genes, 11(8), 885.

Takei, K., Yamaya, T., \& Sakakibara, H. (2004). Arabidopsis CYP735A1 and CYP735A2 Encode Cytokinin Hydroxylases That Catalyze the Biosynthesis of trans-Zeatin. Journal of Biological Chemistry, 279(40), 41866-41872. https://doi.org/10.1074/jbc.M406337200

Wang, P., Zhao, Y., Li, Z., Hsu, C.-C., Liu, X., Fu, L., Hou, Y.-J., Du, Y., Xie, S., Zhang, C., Gao, J., Cao, M., Huang, X., Zhu, Y., Tang, K., Wang, X., Tao, W. A., Xiong, Y., \& Zhu, J.-K. (2018). Reciprocal Regulation of the TOR Kinase and ABA Receptor Balances Plant Growth and Stress Response. Molecular Cell, 69(1), 100-112.e6. https://doi.org/10.1016/j.molcel.2017.12.002

Wang, X., Ding, J., Lin, S., Liu, D., Gu, T., Wu, H., Trigiano, R. N., McAvoy, R., Huang, J., \& $\mathrm{Li}, \mathrm{Y}$. (2020). Evolution and roles of cytokinin genes in angiosperms 2: Do ancient CKXs play housekeeping roles while non-ancient CKXs play regulatory roles? Horticulture Research, 7(1), 29. https://doi.org/10.1038/s41438-020-0246-z

Xiong, Y., McCormack, M., Li, L., Hall, Q., Xiang, C., \& Sheen, J. (2013). Glucose-TOR signalling reprograms the transcriptome and activates meristems. Nature, 496(7444), 181-186. https://doi.org/10.1038/nature12030

Yoshida, S., Mandel, T., \& Kuhlemeier, C. (2011). Stem cell activation by light guides plant organogenesis. Genes \& Development, 25(13), 1439-1450. https://doi.org/10.1101/gad.631211

Zatloukal, M., Gemrotová, M., Doležal, K., Havlíček, L., Spíchal, L., \& Strnad, M. (2008). Novel potent inhibitors of $A$. thaliana cytokinin oxidase/dehydrogenase. Bioorganic \& Medicinal Chemistry, 16(20), 9268-9275. https://doi.org/10.1016/j.bmc.2008.09.008

Zürcher, E., Tavor-Deslex, D., Lituiev, D., Enkerli, K., Tarr, P. T., \& Müller, B. (2013b). A Robust and Sensitive Synthetic Sensor to Monitor the Transcriptional Output of the Cytokinin Signaling Network in Planta. Plant Physiology, 161(3), 1066-1075. https://doi.org/10.1104/pp.112.211763

\section{Acknowledgements}

We thank Sebastian Wolf and Aurelio Teleman for their ideas and scientific input and Tomas Werner for sharing the p35S:cMyc-CKX1 line. The authors give sincere thanks to Hana Martínková and Petra Amakorová for their help with phytohormone analyses. This work was supported by the ERC grant (\#282139) "StemCellAdapt", the CellNetworks Cluster of Excellence (DFG) and the SFB873 (DFG) to JL, as well as the Ministry of Education, Youth 
and Sports of the Czech Republic (European Regional Development Fund-Project "Plants as a tool for sustainable global development” No. CZ.02.1.01/0.0/0.0/16_019/0000827).

\section{Author contributions}

O.N. and M.S. performed the cytokinin profiling experiment, Y.D. performed the ribosome fractionation experiment and gave input for results interpretation, L.A.R. gave input for results interpretation, A.P. performed one WUS reporter assay generated the cross between the DR line and p35S ARR12DDK:GR and designed experiments, D.J. performed all other experiments, designed experiments, curated and analyzed the data, wrote the first draft, revised and edited the manuscript, J.U.L. designed experiments, wrote the final manuscript and acquired the funding. 


\section{Figures and legends}

a

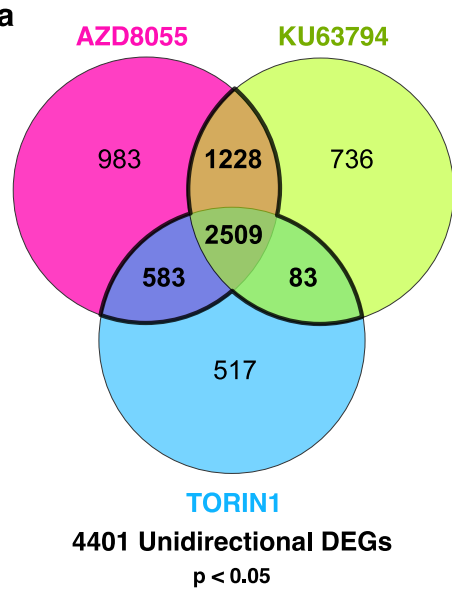

C

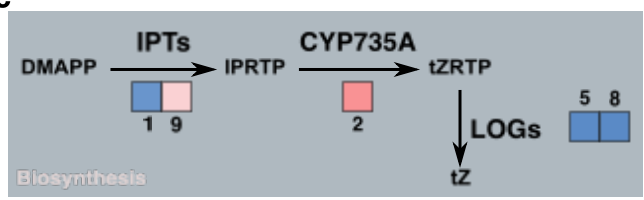

CK
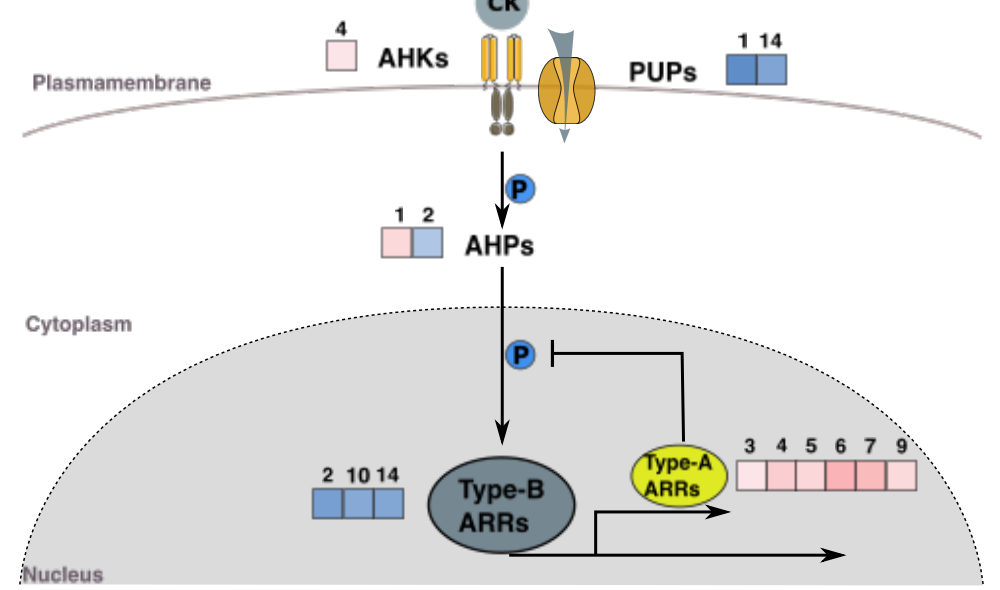

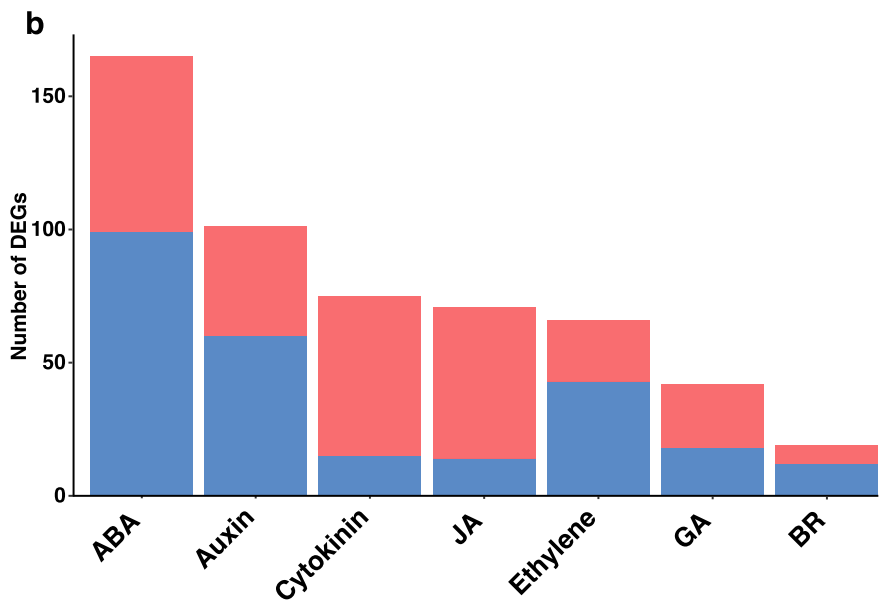

Figure 1: TOR inhibition leads to reduced CK signaling in the SAM

a) Venn diagram of differentially expressed genes obtained from RNAseq of shoot tissue from 4 day old seedlings treated with different TOR inhibitors for $8 \mathrm{~h}$. b) Gene count of DEGs from RNAseq analysis annotated with hormone signaling function from $G O$ term analysis. $A B A=$ abscisic acid, JA = jasmonic acid, GA = gibberellic acid, BR = brassinosteroids. c) Schematic representation of the CK signaling pathway. Color code represents log fold change value obtained from RNAseq analysis. The numbers over the boxes indicate the isoform number of the respective gene. IPT = isopentyltransferase, $L O G=$ lonely guy, $A H K=$ Arabidopsis histidine kinase, $\mathrm{AHP}=$ Arabidopsis histidine phosphotransferase, $\mathrm{ARR}=$ Arabidopsis response regulator, PUP = purine permease. $\mathbf{d}$, e) Representative microscopic image of pTCSn:GUS reporter line treated with either DMSO or AZD8055 for $24 \mathrm{~h}$. Scale bar $=30 \mu \mathrm{m}$. 


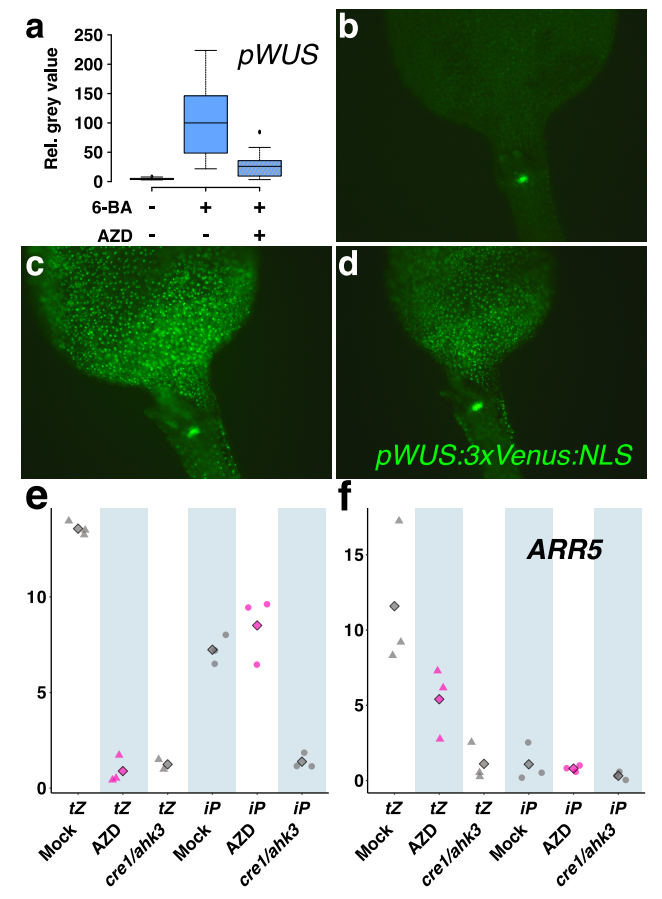

Figure 2: CK output potential is not affected by TOR inhibition

a) Quantification of pWUS:3xVenus:NLS reporter signal from 5 day old etiolated seedlings treated with $0.5 \mu \mathrm{M}$ 6-benzyladenine and $2 \mu \mathrm{M}$ AZD8055 for 3 days. b-d) Representative microscopic images of p35S:ARR12DDK:GR crossed with pWUS:3xVenusNLS. 3 day old light grown seedlings were treated with $10 \mu \mathrm{M}$ dexamethasone and $2 \mu \mathrm{M}$ AZD8055 for $24 \mathrm{~h}$.

e, f) Relative expression values, normalized to PP2A obtained with q-RT-PCR from e) root and the respective f) shoot tissue are shown. 4 day old seedlings preincubated on AZD8055 or mock were sprayed with either $100 \mathrm{nM}$ trans-Zeatin (tZ) or $100 \mathrm{nM}$ isopentyladenine (iP) solution. Data points show expression values from biological replicates (triangles and circles) together with the calculated mean (rhombus). 

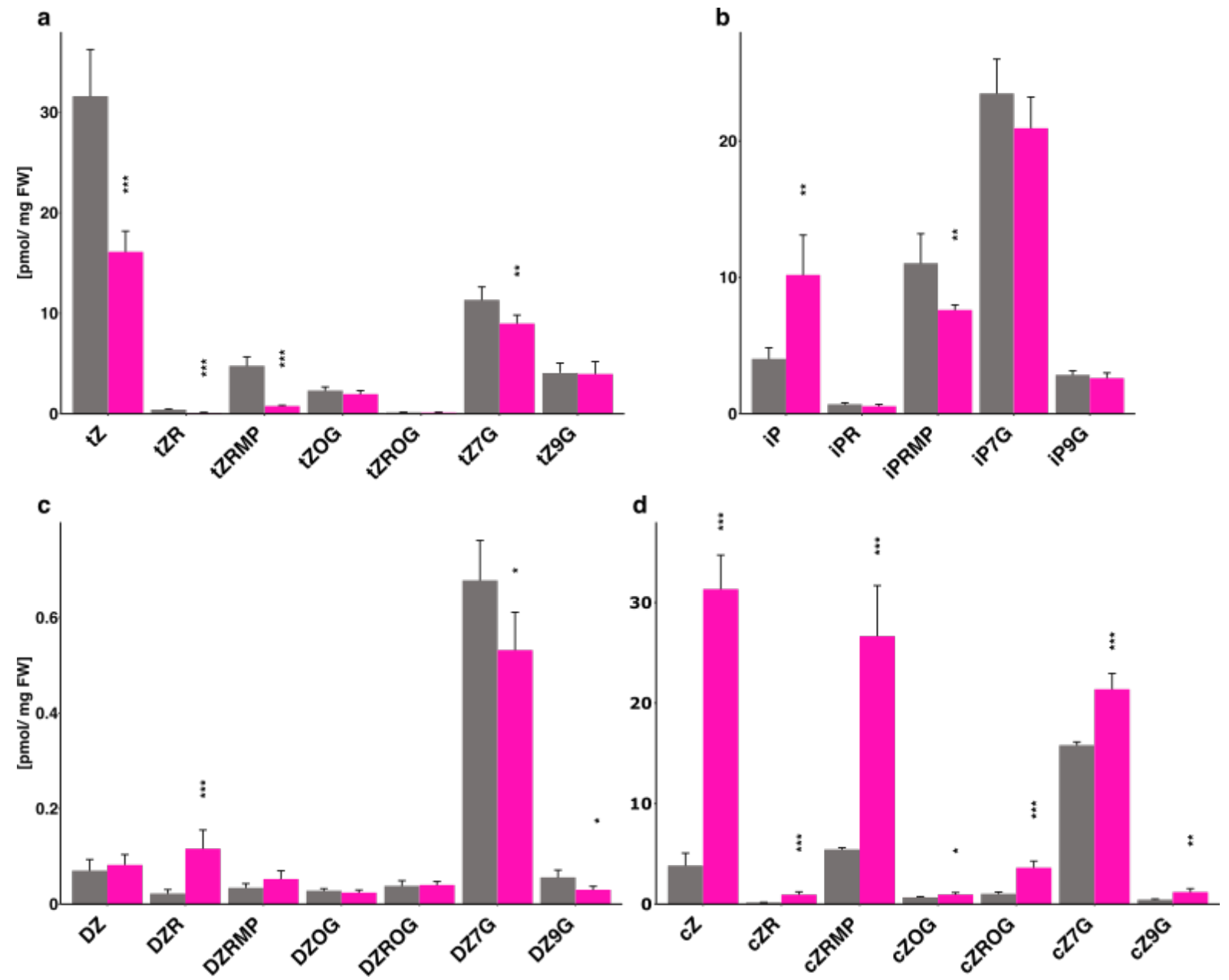

Figure 3: Disturbed CK homeostasis after TOR inhibition

Quantification of different CK compounds in 4 day old seedlings treated with AZD8055 (magenta) or mock (grey) for $8 \mathrm{~h}$. Asterisks indicated significant differences of the AZD8055 treated sample compared with the respective mock calculated with paired Student's t-test ${ }^{*}$, ${ }^{* *}$, and ${ }^{* * *}$ correspond to P-values of $0.05>p>0.01,0.01>p>0.001$, and $p<0.001$, respectively). Error bars indicate standard deviation. $n=5$. a) $t Z=$ trans-zeatin, $t Z R=$ transzeatin riboside, $\mathrm{tZRMP}=$ trans-zeatin riboside-5'monophosphate, $\mathrm{tZOG}=$ trans-zeatin $\mathrm{O}$ glucoside, $\mathrm{tZROG}=$ trans-zeatin riboside O-glucoside, $\mathrm{tZ7G}=$ trans-zeatin-7-glucoside, $\mathrm{tZ9G}$ $=$ trans-zeatin-9-glucoside $\mathbf{b}) \mathrm{iP}=$ isopentyladenine, $\mathrm{iPR}=$ isopentyladenosine, $\mathrm{iPRMP}=$ isopentyladenosine-5'monophosphate, $\mathrm{iP7G}=$ isopentyladenine-7-glucoside, $\mathrm{iP9G}=$ isopentyladenine-9-glucoside $\mathbf{c}$ ) $\mathrm{DZ}=$ dihydrozeatin, $\mathrm{DZR}=$ dihydrozeatin riboside, $\mathrm{DZRMP}=$ dihydrozeatin riboside-5'monophosphate, DZOG = dihydrozeatin O-glucoside, DZROG = dihydrozeatin riboside O-glucoside, $\mathrm{DZ7G}=$ dihydrozeatin-7-glucoside, $\mathrm{DZ9G}=$ dihydrozeatin-9-glucoside $\mathbf{d}) \mathrm{cZ}=$ cis-zeatin, $c Z R=$ cis-zeatin riboside, $c Z R M P=$ cis-zeatin riboside-5'monophosphate, $c Z O G=$ cis-zeatin O-glucoside, $c Z R O G=$ cis-zeatin riboside Oglucoside, $c Z 7 G$ = cis-zeatin-7-glucoside, $c Z 9 G$ = cis-zeatin-9-glucoside 

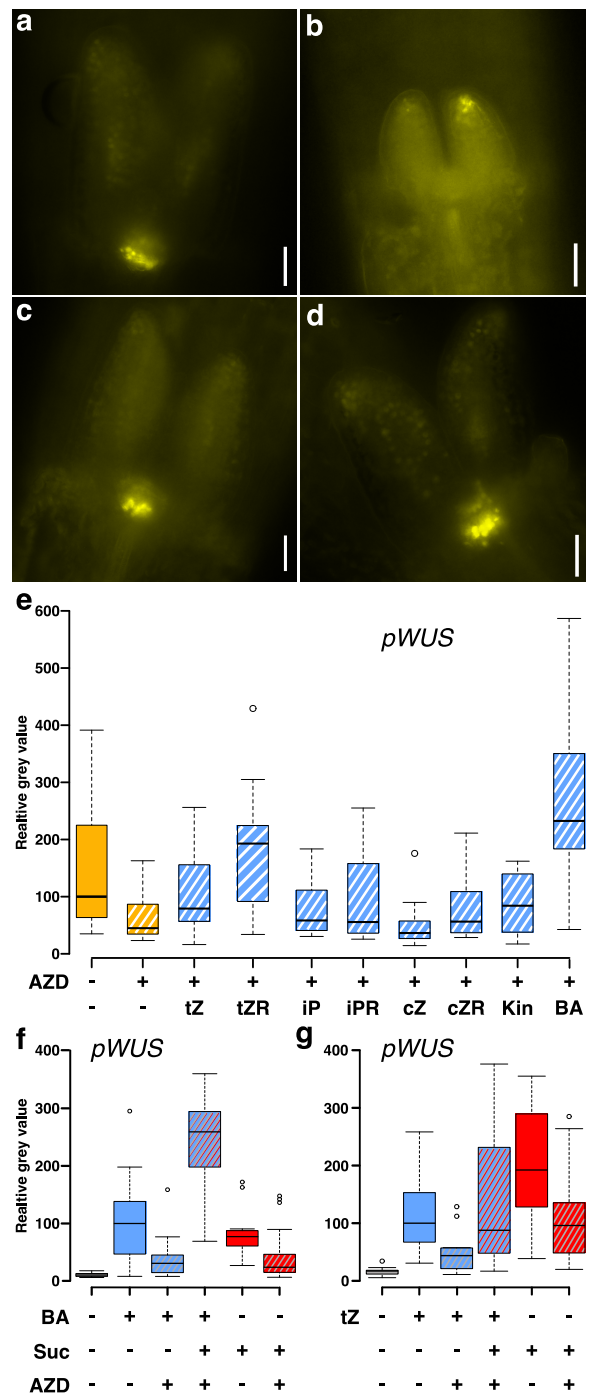

Figure 4: trans-Zeatin rescues WUS expression upon TOR inhibition

Representative microscopic images pWUS:3xVenus:NLS reporter line treated with a) mock b) $2 \mu$ AZD8055 c) AZD + $0.5 \mu \mathrm{M}$ tZR and e) AZD + $0.5 \mu \mathrm{M}$ 6-BA. Images were acquired as black and white and false color coded with imagej. Scale bar $=30 \mu \mathrm{m}$. e) Quantification of pWUS:3xVenus:NLS reporter signal. 3 day old seedlings grown in the light were treated with $2 \mu \mathrm{M}$ AZD8055 and $0.5 \mu \mathrm{M}$ of different CK derivatives for 1 day. $\mathrm{tZ}=$ trans-Zeatin, $\mathrm{tZR}=$ transZeatin riboside, $\mathrm{iP}=$ isopentyladenine, $\mathrm{iPR}=$ isopentyladenosine, $\mathrm{cZ}=$ cis-Zeatin, $\mathrm{cZR}=$ cisZeatin riboside, $\mathrm{Kin}=$ kinetin, $\mathrm{BA}=6$-benzyladenine. $\mathrm{p}$-values were calculated comparing $\mathrm{CK}$ supplemented conditions with only AZD treated condition. $t Z(p=0.019)$, $t Z R(p=0.00006)$ and 6-BA $(p=0.0003)$ were found significantly different using paired t-test. $n=13-20 . \mathbf{f}, \mathbf{g})$ Quantification of pWUS:3xVenus:NLS reporter signal. 2 day old etiolated seedlings were treated with $0.5 \mu \mathrm{M}$ 6-benzyladenine or trans-Zeatin, $2 \mu \mathrm{M}$ AZD8055 and/or 1\% sucrose for 3 days in the dark. f) $n=13-30$ g) $A Z D+t Z+s u c$ is significantly different from $A Z D+t Z$ $(p=0.028$, paired t-test) $n=12-20$. 

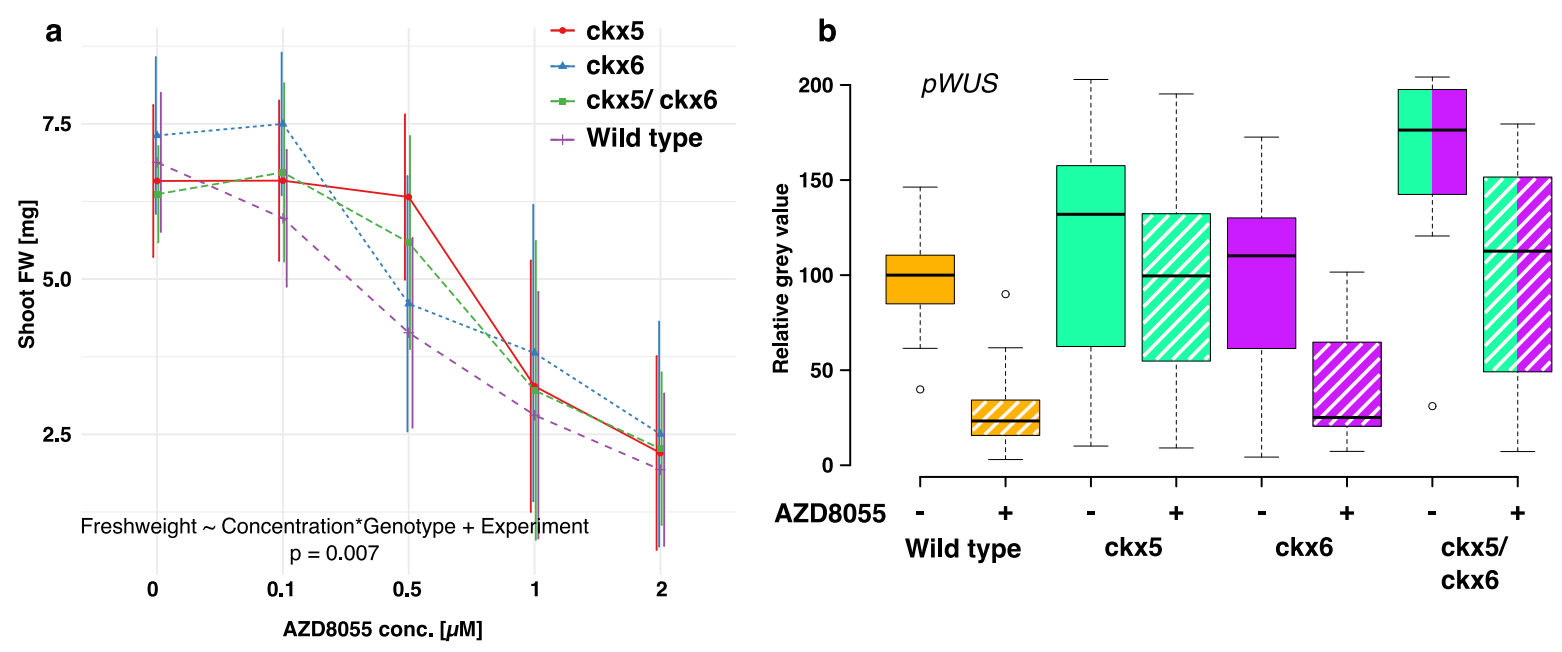

C

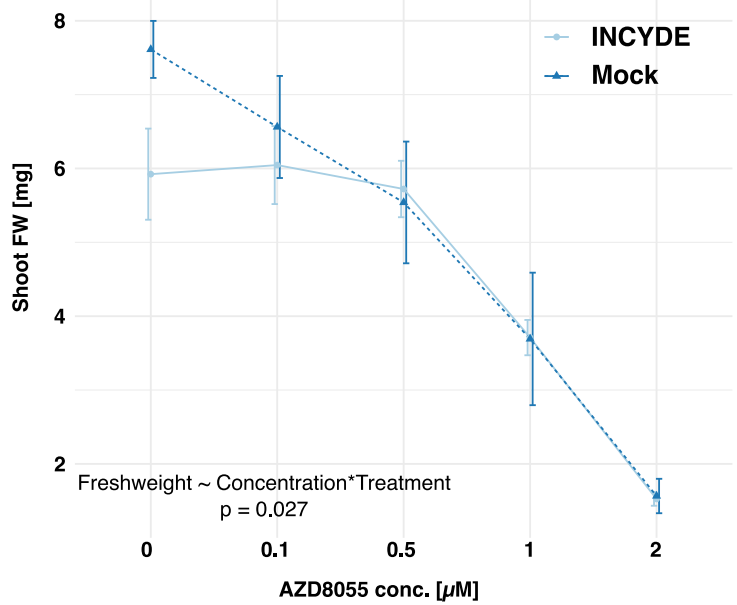

d

e
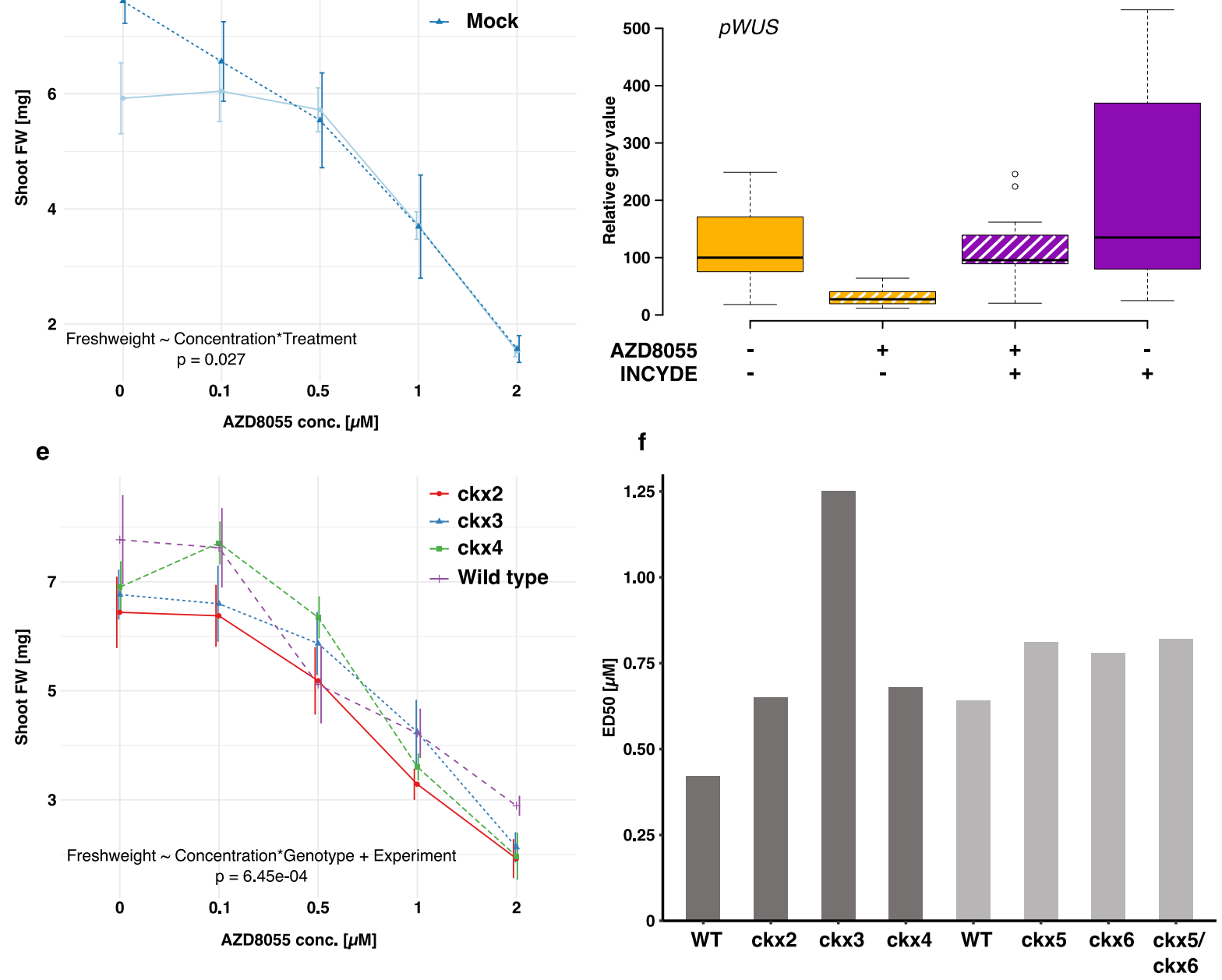

Figure 5: $C K X$ mutants are resistant to TOR inhibition

a, c, e) Average shoot fresh weight per seedling was calculated from measurements of $\sim 40$ seedlings in batches of 5-10, that were transferred at 4 DAG to different concentrations of AZD8055 for 7 days. Error bars represent standard deviation. The indicated interaction terms were tested with mixed ANOVA using pooled data from two independent experimental 
repetitions (except c)). a) $c k x 5, c k x 6$ and $c k x 5 / 6$ are in the genetic background of pWUS:3xVenusNLS, pCLV3:mCherry:NLS and were tested against this background. c) 75 nM INCYDE. b, d) Quantification of pWUS:3xVenus:NLS reporter signal. Seedlings were grown in the light for 3 days and treated with mock, $2 \mu \mathrm{M}$ AZD8055 and/ or 75 nM INCYDE for 1 day. f) ED50 values for different CKX mutant lines. Colors indicate different genetic background. Dark grey = col-0; light grey = pWUS:3xVenusNLS, pCLV3:mCherry:NLS. 


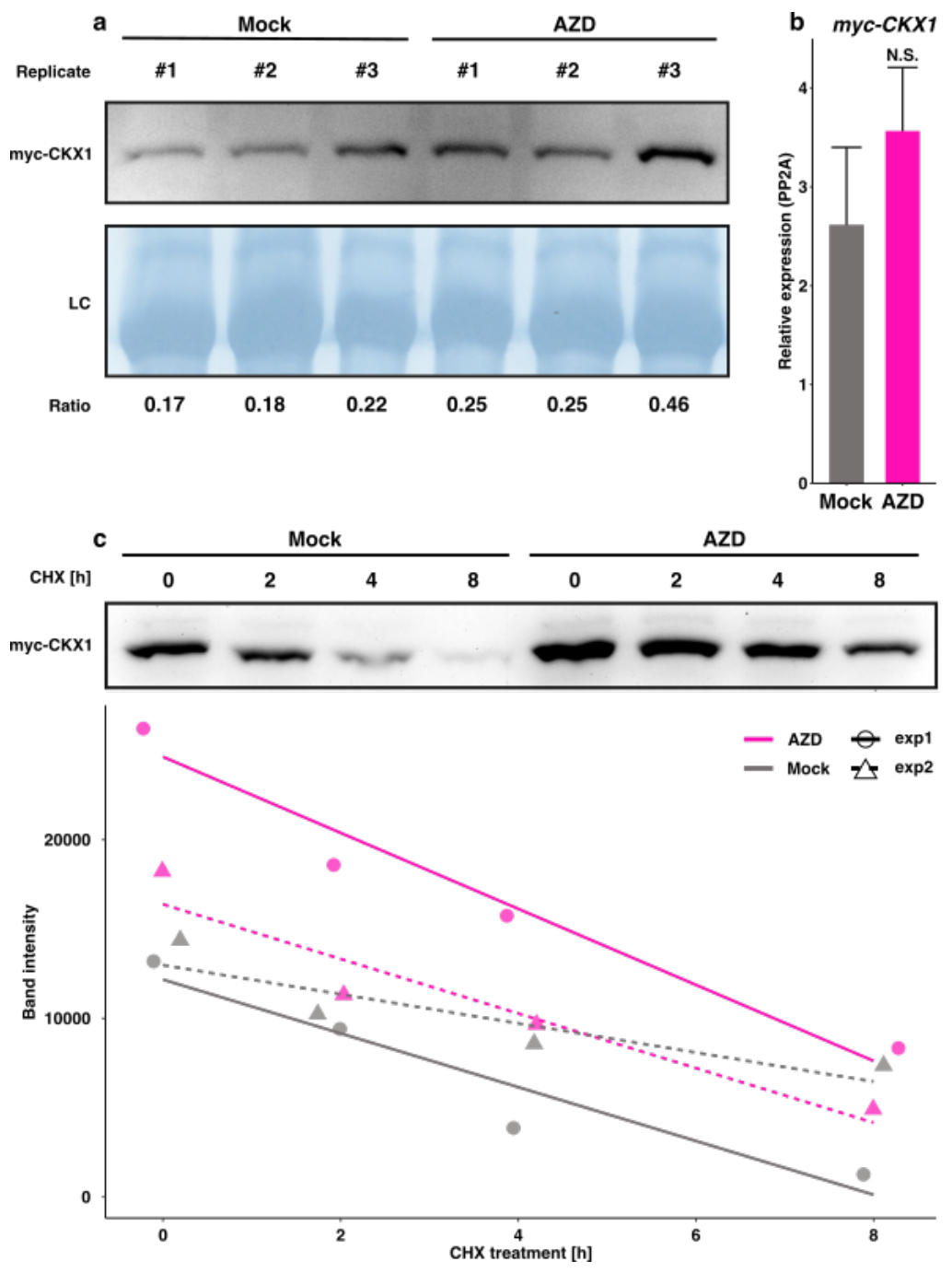

Figure 6: cMyc-CKX1 protein accumulates upon TOR inhibition

a) Western blot of p35S:cMyc-CKX1 protein extract probed with anti cMyc serum. Replicates are from three independent experimental repetitions. Loading control (LC) stained with amido black. Ratios were calculated between band intensities from myc-CKX1 signal and the respective LC. b) q-RT-PCR of p35S:cMyc-CKX1 seedlings with primers against the ectopic transcript. No significant difference using two biological replicates was found using paired ttest $(p=0.3)$. Error bars represent standard deviation. $c)$ Western blot of p35S:cMyc-CKX1 protein extract probed with anti cMyc serum. Seedlings were pre-incubated on AZD8055 and then flooded with $200 \mu \mathrm{M}$ cycloheximide solution for the indicated time. Band intensities from two independent experimental repetitions were plotted with linear regression lines. 

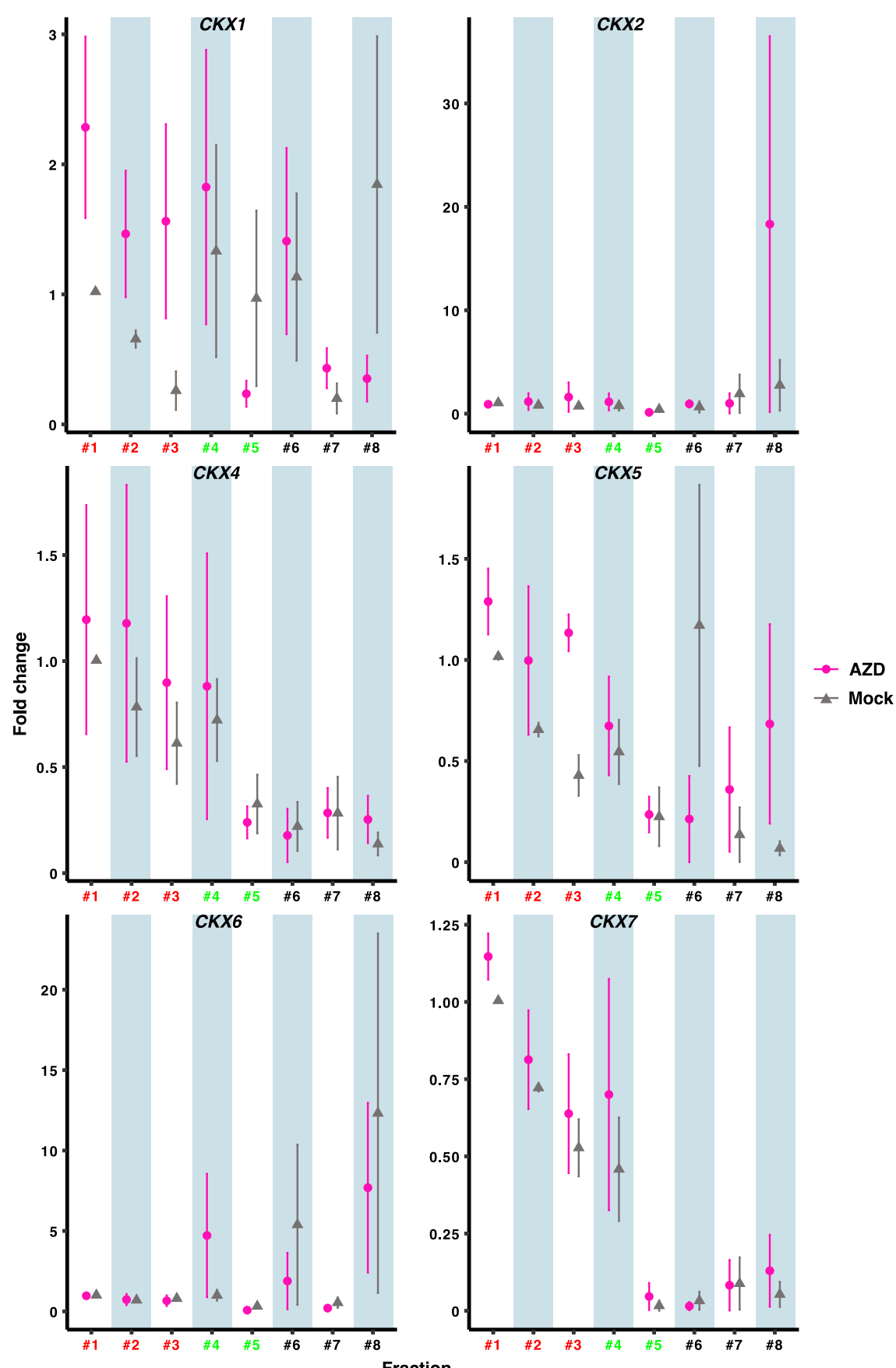

Figure 7: Transcripts of CKX isoforms accumulate in heavy polysomal fractions

CKX transcripts were quantified in ribosomal fractionations by q-RT-PCR relative to $U B / 10$ and normalized to the respective mock of fraction \#1. Error bars represent standard error of the mean of data pooled from three independent experimental repetitions (except CKX2+7, $\mathrm{n}=2$ ). Red numbers indicate heavy polysomal fractions (fractions 1-3), green numbers light polysomal fractions (fractions 4+5) and black number monosomal fractions (fractions 6-8). Individual data points are shown in the supplements (Fig. S7). 